\title{
Measuring Regularity in Daily Behavior for the Purpose of Detecting Alzheimer
}

\author{
Saskia Robben \\ Amsterdam University of \\ Applied Sciences \\ Wibautstraat 2-4 \\ $1091 \mathrm{GM}$, Amsterdam, \\ Netherlands \\ s.m.b.robben@hva.nl
}

\author{
Ahmed Nait Aicha \\ Amsterdam University of \\ Applied Sciences \\ Wibautstraat 2-4 \\ 1091 GM, Amsterdam, \\ Netherlands \\ a.nait.aicha@hva.nl
}

\author{
Ben Kröse \\ University of Amsterdam \\ Science Park 904 \\ 1098 XH Amsterdam, \\ Netherlands \\ b.j.a.krose@uva.nl
}

\begin{abstract}
This paper presents a study of sensor data from a person who developed Alzheimer's disease during a 4-year monitoring period and who is monitored with simple ambient sensors in her home. Our aim is to find data analysis methods that reveal relevant changes in the sensor pattern that occur before the diagnosis. We focus on the quantification of regularity, which is identified as a relevant indicator for the assessment of a disease such as Alzheimer's. Two unsupervised methods are studied. Restricted Boltzmann Machines are trained and the resulting weights are visualized to see whether there are changes in regularity in the behavioral pattern. Fast Fourier Transformation is applied to the sensor data and the spectral characteristics are determined and compared with the same purpose. Both methods reveal changes in the pattern between different periods. Both methods therefore are useful in quantifying and understanding changes in the regularity of the daily pattern.
\end{abstract}

\section{Keywords}

Ambient Assisted Living, Sensor monitoring, Signal processing

\section{INTRODUCTION}

Lifestyle monitoring systems that use simple ambient sensors in the home have been presented to detect deviant patterns in the behavior [13, 1]. Not all deviant patterns in behavior are relevant, the challenge lies in identifying the changes that can be connected to a change in health. Many (commercially available) systems implement rule-of-thumb thresholds and adjust these in collaboration with the people in the network of the elderly to minimize false alarms. More informed approaches exist, for example methods that try

Permission to make digital or hard copies of all or part of this work for personal or classroom use is granted without fee provided that copies are not made or distributed for profit or commercial advantage and that copies bear this notice and the full citation on the first page. To copy otherwise, to republish, to post on servers or to redistribute to lists, requires prior specific permission and/or a fee.

PervasiveHealth 2016, May 16-19, Cancun, Mexico

Copyright $\odot 2016$ EAI 978-1-63190-051-8 to derive the Activities of Daily Living (ADL) [8] or other activities from the sensor data $[4,16]$.

Besides recognizing ADL, there is much evidence that regularity of the daily pattern is very informative for assessing the health status. Changes in behavioral circadian rhythms are associated with aging [7] and detecting shifts in the $24 \mathrm{~h}-$ rhythm is useful [5] and can be related to physical functioning [11]. Sleep patterns and patterns when leaving the house [9] can be measured, and similar metrics are shown to be useful in monitoring cognitive decline or Alzheimer [10]. Therefore, in this paper we focus on measuring the regularity of the behavior of independent living older adults.

We present two unsupervised methods that help to understand the regularity of the sensor signal and can quantify it. First, Restricted Boltzmann Machines (RBM) are applied as a type of autoencoder. Because the intrinsic dimension for a regular daily pattern is low, autoencoders can be used to make a non-linear projection of the high-dimensional sensor data to a lower dimensionality. Using RBM's as a singlelayered autoencoder, visualizations of the weights provide insight in regularity of the participant. Secondly, the spectral distribution of the periodic data is explored with a Fast Fourier Transformation (FFT). Under the hypothesis that a regular temporal pattern shows a more peaked distribution in the frequency domain than a non-regular temporal pattern we studied periodic and entropy characteristics from this distribution. Both methods are used to inspect changes in regularity of the daily pattern of our participant.

For the illustration and validation of our approach, we draw upon our monitoring data set in which we collected longitudinal sensor data $[14,12]$. In this paper we use the data of a resident who developed Alzheimer during a four year period in which her behavior and health were monitored. We first describe the data and approach, end then show the results of our methods in this specific case.

\section{DATA}

The data comes from a larger set that is collected in the homes of 23 independent living older adults. All participants have a sensor system in their home that consists of approximately 16 binary sensors, mostly motion sensors to cover all major locations and contact switches on doors. See Figure 1 for the apartment of the case described in this paper. Every three months the participants are visited to take health assessments and questionnaires (see [15] for an overview of 


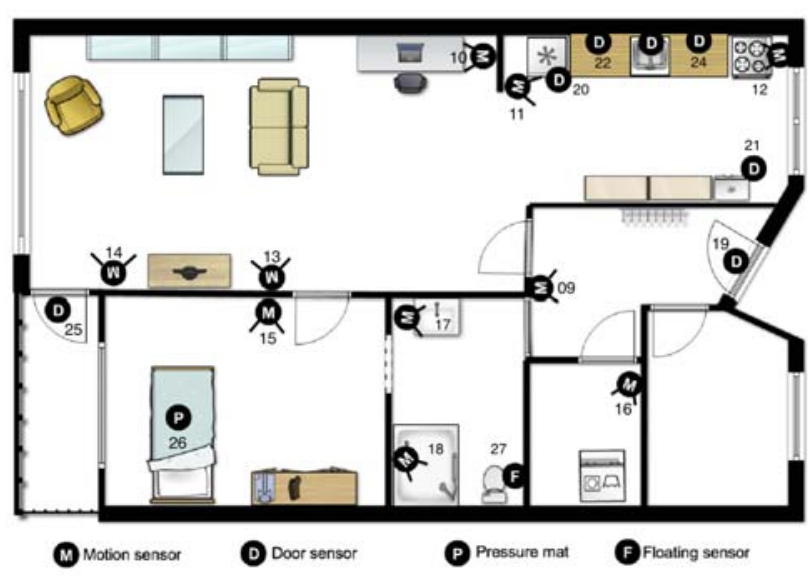

Figure 1: Floor plan of the apartment. ' $\mathrm{M}$ ' indicates a motion sensor and 'D' (door) indicates a contact switch.

the metrics). For this paper the data were selected of a participant who developed Alzheimer's disease during the measurement period.

\subsection{Data representation}

From the raw sensor data we derived two representations. The binary location representation gives the participant's presence in different locations (such as bedroom, bathroom and outside) with precision of a minute. For every day this results in a set of daily binary feature vectors of dimension $D=\#$ minutesperday $=1440$. Figure 2 visualizes all the sensor data of our participant using this representation. The location duration representation gives the amount of time that a participant is present in a location in minutes per hour. For every day this results in a set of daily feature vectors of dimension $D=$ \#hoursperday $=24$. An example of the bedroom signal of a week is given in Figure $4 \mathrm{a}$.

\subsection{Case characteristics}

The person in our case study is a 87 year old female who was monitored for four years. When monitoring started, she was very independent, only having a housekeeper. At first she was stable, no incidents were reported. She had several (social) activities outside her house. She had some problems with her joints and used a walker (outside). She did exercised in the morning, also in her bed. In March 2014, after 2.5 years of monitoring, it was reported that she was diagnosed with Alzheimer. She started going to day care twice a week soon after, which is visible as a change of color in Figure 2. In the beginning we see some changes in patterns. This can be explained by the adjustment of sensors in the home. At first, the living room was not fully captured, therefore the tracking algorithm missed sometimes presence in the living room.

\subsection{Data selection}

Data from two different periods of 30 days were selected for comparison. One period, referred to as the period with Alzheimer, is just before diagnosis in March 2014. This is because after the diagnosis, some immediate interventions were started that altered the pattern. The assumption here is that a change (probably) took place before actual diagnosis. Another period, without Alzheimer, is approximately

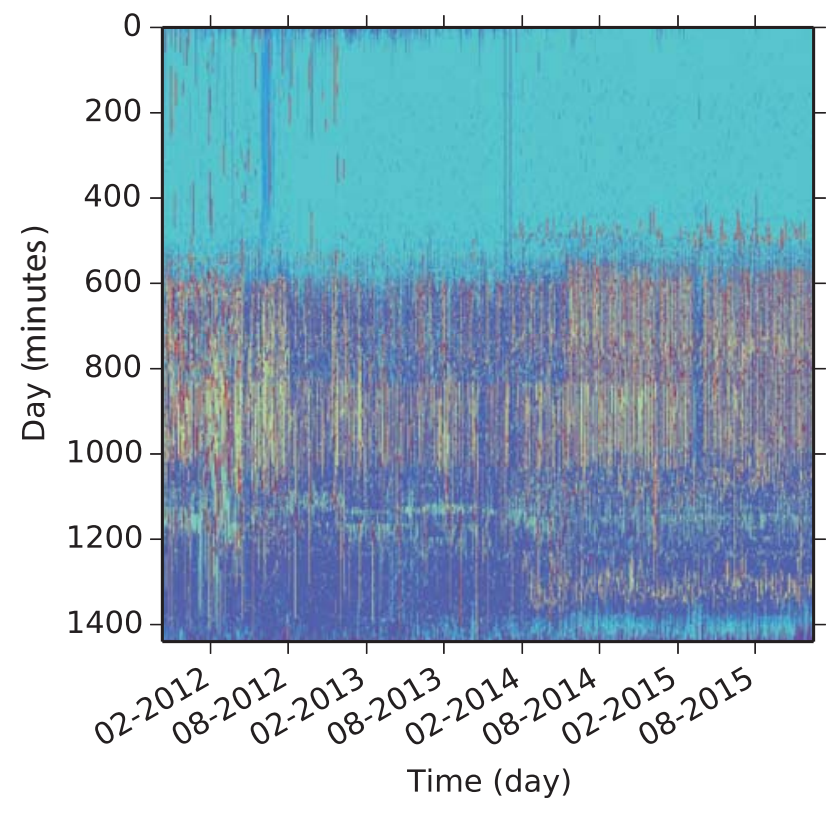

Figure 2: Four years of monitoring data. Each color represents the location of the resident in a specific time slot (precision of a minute). The $\mathrm{x}$ axis shows the day of measurement. The y axis displays the time of day, currently provided in minutes in the day (from midnight at the top until midnight again at the bottom).

a year before diagnosis (March 2013). We selected a year earlier to minimize seasonal effects.

\section{APPROACH}

The two methods (RBM and FFT) that are used to compare differences in regularities of the daily pattern between the two periods (with or without Alzheimer) are introduced below.

\subsection{Restricted Boltzmann Machines}

Restricted Boltzmann Machines are two-layered networks, suited for modeling binary data. The RBM consists of a visible layer of which the units corresponds to the input and a hidden layer of which the units are generally interpreted as feature detectors. No connections within these layers are allowed, but between the layers the network is fully connected. A trained network can be regarded as a type of auto-encoder, where the dimensionality reduction is determined by the size of the hidden layer. The weights represent the features that describe the data best

The size of the hidden layer is kept limited, because we are mainly interested in the general pattern. The number of hidden units in our experiments is limited to four, because adding more hidden units caused a (visual) redundancy in features. We expect to detect features that represent typical days (i.e. similar as in PCA where the first few principal components represent general trends and less frequent patterns are represented in the later components.)

Existing Theano implementations $[2,3]$ were adjusted to perform the experiments. Two separate RBM's are trained [6] with the binary location representation as input, for the 

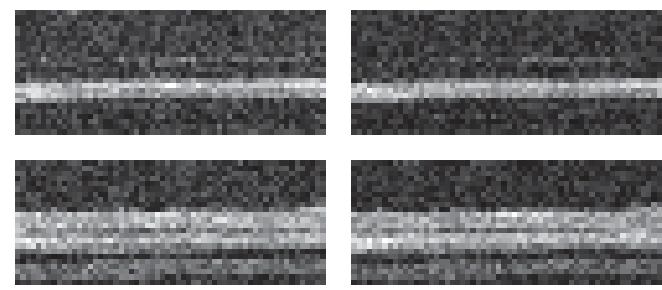

(a) Without Alzheimer
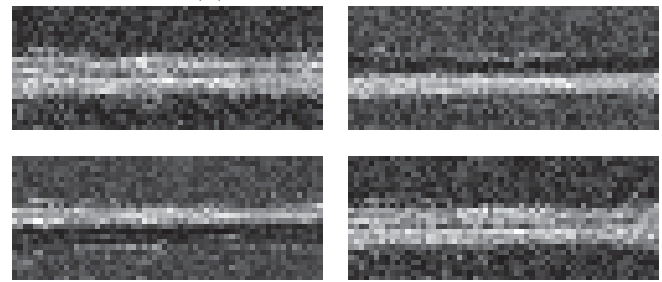

(b) With Alzheimer

Figure 3: Visualized weights of two RBM's trained on outside presence data. Each figure displays the weights to each of the four hidden units in a $2 \mathrm{x} 2$ arrangement. The weights to each hidden unit are reshaped to a $60^{*} 24$ matrix for interpretation purposes.

two periods (with and without Alzheimer). The weights of each are visualized to get insight in the typical days that are present in that period. This experiment was repeated for each of the locations separately.

\subsection{Fast Fourier Transformation}

Fast Fourier Transformation is a widely used method to filter signals. Given a time series signal $X(t)$, the FFT decomposes it into sine waves (with frequencies $\omega$ ) that together compose the signal. Thus, the FFT is a transformation of an function in a time domain $X(t)$ into a function in a frequency domain $Y(\omega)$. The representation of the signal in the frequency domain allows us to calculate some spectral characteristics of the sine waves such as the periodicity, the amplitude. Additionally, the spectral entropy is calculated using the equation: $E=-\sum_{i=1}^{n}\left(p_{i} \log \left(p_{i}\right)\right)$, where $p_{i}$ is the normalized squared spectral density of frequency $i$, and $n$ is the total number of frequencies. In our case, the input function of the FFT is the duration-location representation of the sensor data as described in Section 2.1. The analysis is performed for each of the locations separately. An example of a such input function for the 'bedroom' location during one week and its corresponding frequency function is given in Figure 4.

\section{RESULTS}

RBM. The weights between the visible and the four hidden layer are visualized to get insight in the regularity of the data. To get useful visualizations, the weights of the visible layer to the hidden layer are visualized per hidden unit. These weights are then transformed to a $24 * 60$ matrix, so each row is an hour (of 60 minutes), starting from midnight at the top.

The results that were most clearly visible were obtained with data where the person is outside (away from home). Figure 3 shows these weights for the two different periods. All weights display a clear circadian rhythm, it is easy to dis-

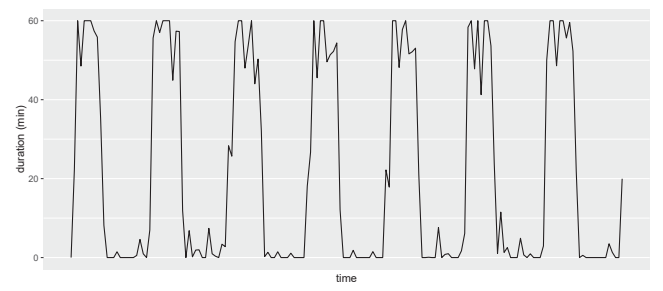

(a) Sample of the bedroom signal in the time domain.

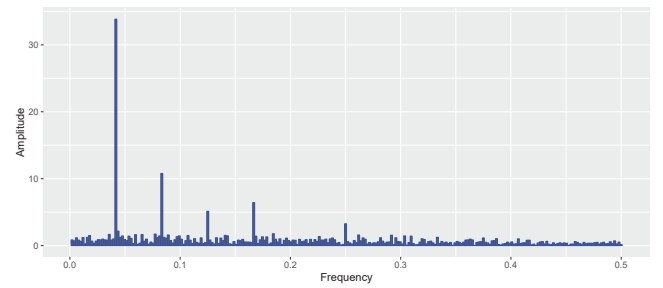

(b) The corresponding representation in the frequency domain.

Figure 4: The representation of the 'bedroom' signal in both the time domain and the frequency domain.

criminate between night (approx upper half) and day (lower half). But the pattern changes between periods. Additionally, the weights in the figure with Alzheimer are less extreme than the weight pattern in the figure without Alzheimer, indicating a less regular pattern.

FFT. The results are given in Table 1. The table shows the spectral characteristics per location for both the periods without and with the Alzheimer diagnosis. These spectral characteristics are obtained by applying FFT to convert the signals in the time domain into frequencies in the frequency domain for each location. For example, converting the bedroom signal from the time domain into the frequency domain results in Figure 4. The results in Table 1 show an increase of the spectral entropy in all the locations. This is a consequence of a more noisy signal in the period with Alzheimer compared to the period without the diagnosis. More likely, the resident has less regularity in her activities in the period with the diagnosis. Another interesting finding is the increase of the periodicity of the dominant frequency in the 'bathroom'-location from 12 hours (twice a day) to 24 hours. This may be an indication that the subject sometimes forgets to do some bathroom-activity. Note that both the 'outside' and 'laundry' locations have several dominant frequencies (graphs not included). Therefore, no conclusion can be drawn from the decrease of the periodicity of the dominant frequency for these two locations.

\section{DISCUSSION AND CONCLUSION}

It is a promising finding that the differences in the behavior of the participant can be captured by both the filters in a RBM and the FFT. Both methods display changes in the pattern before and after the diagnosis.

Sensor systems enable early diagnosis and intervention in the case of Alzheimer, but there is no clear protocol on what features to focus. Regularity is a promising candidate feature, and this paper provides directions for appropriate tools to quantify regularity. Both the FFT and RBM approach can act as tools to understand and explore the data. And the fact that both methods can be used for dimensionality 


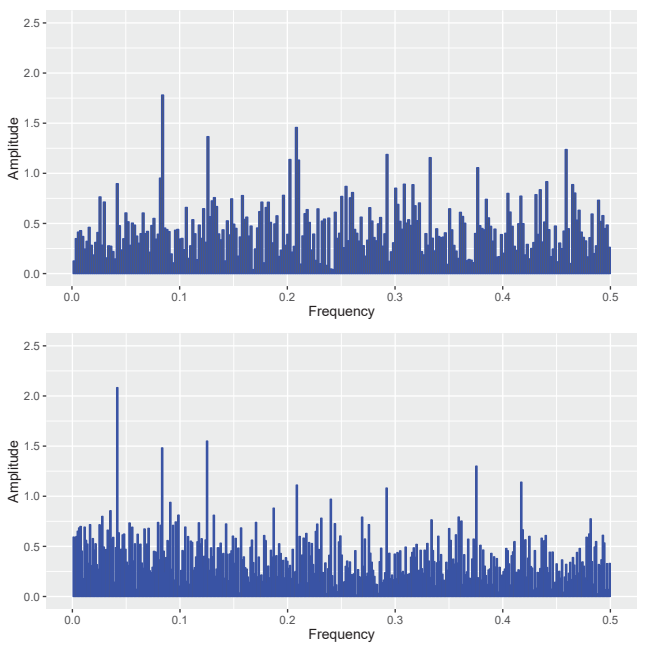

Figure 5: The representation of the bathroom signal in the frequency domain using the sensor data collected in the periods with (upper graph) and without (lower graph) Alzheimer.

\begin{tabular}{r|rr|rr|rr} 
Location & \multicolumn{2}{|c|}{ Periodicity } & \multicolumn{2}{|c|}{ Amplitude } & \multicolumn{2}{c}{ Entropy } \\
\hline bedroom & 24.05 & 24.00 & 33.63 & 30.41 & 2.02 & 2.51 \\
kitchen & 24.75 & 24.44 & 2.83 & 3.07 & 5.32 & 5.51 \\
livingroom & 24.75 & 25.23 & 11.60 & 5.01 & 4.01 & 4.57 \\
bathroom & 12.15 & 23.97 & 1.06 & 2.08 & 5.67 & 6.06 \\
laundry & 147.33 & 83.33 & 0.13 & 0.15 & 5.44 & 6.02 \\
outside & 491.00 & 129.00 & 3.06 & 2.70 & 5.07 & 5.74
\end{tabular}

Table 1: Spectral characteristics calculated for both the periods WITHOUT (first column) and WITH (second column) Alzheimer. Shown are the periodicity (hours) and the amplitude (minutes) of the dominant frequency. Also, the entropy of the signal in the frequency domain is calculated.

reduction, this is promising for modeling Alzheimer. When more longitudinal data is available to train models, these tools can be used to successfully deploy sensors in the home to automatically detect Alzheimer in an early stage.

\section{ACKNOWLEDGMENTS}

We thank our study participants, collaborators and colleagues, particularly Margriet Pol. This research was supported by RVO/Agentschap NL (FIT and Health-lab) and SIA (Hipper and Smart Systems for Smart Services).

\section{REFERENCES}

[1] H. Alemdar and C. Ersoy. Wireless sensor networks for healthcare: A survey. Computer Networks, 54(15):2688-2710, 2010.

[2] F. Bastien, P. Lamblin, R. Pascanu, J. Bergstra, I. J. Goodfellow, A. Bergeron, N. Bouchard, and Y. Bengio. Theano: new features and speed improvements. Deep Learning and Unsupervised Feature Learning NIPS 2012 Workshop, 2012.

[3] J. Bergstra, O. Breuleux, F. Bastien, P. Lamblin, R. Pascanu, G. Desjardins, J. Turian,

D. Warde-Farley, and Y. Bengio. Theano: a CPU and
GPU math expression compiler. In Proceedings of the Python for Scientific Computing Conference (SciPy), June 2010. Oral Presentation.

[4] L. Chen, J. Hoey, C. D. Nugent, D. J. Cook, and Z. Yu. Sensor-based activity recognition. Systems, Man, and Cybernetics, Part C: Applications and Reviews, IEEE Transactions on, 42(6):790-808, 2012.

[5] C. Franco, J. Demongeot, C. Villemazet, and N. Vuillerme. Behavioral telemonitoring of the elderly at home: Detection of nycthemeral rhythms drifts from location data. In IEEE 24 th International Conference on Advanced Information Networking and Applications Workshops (WAINA), pages 759-766. IEEE, 2010.

[6] G. Hinton. A practical guide to training restricted boltzmann machines. Momentum, 9(1):926, 2010.

[7] M. A. Hofman and D. F. Swaab. Living by the clock: the circadian pacemaker in older people. Ageing research reviews, 5(1):33-51, 2006.

[8] S. Katz, A. Ford, R. Moskowitz, B. Jackson, and M. Jaffe. Studies of illness in the aged. JAMA: the journal of the American Medical Association, 185(12):914-919, 1963.

[9] A. Kealy, K. McDaid, J. Loane, L. Walsh, and J. Doyle. Derivation of night time behaviour metrics using ambient sensors. In Pervasive Computing Technologies for Healthcare (PervasiveHealth),2013 7th International Conference on, pages 33-40. IEEE, 2013.

[10] B. E. Lyons, D. Austin, A. Seelye, J. Petersen, J. Yeargers, T. Riley, N. Sharma, N. C. Mattek, H. Dodge, K. Wild, et al. Pervasive computing technologies to continuously assess alzheimerŠs disease progression and intervention efficacy. Frontiers in Aging Neuroscience, 7:102, 2015.

[11] J. Merilahti, P. Viramo, and I. Korhonen. Wearable monitoring of physical functioning and disability changes, circadian rhythms and sleep patterns in nursing home residents. IEEE Journal of Biomedical and Health informatics (to appear), 2015.

[12] A. Nait Aicha, G. Englebienne, and B. Kröse. Continuous gait velocity analysis using ambient sensors in a smart home. In Ambient Intelligence, pages 219-235. Springer, 2015.

[13] P. Rashidi and A. Mihailidis. A survey on ambient-assisted living tools for older adults. IEEE journal of biomedical and health informatics, 17(3):579-590, 2013.

[14] S. Robben, G. Englebienne, M. Pol, and B. Kröse. How Is Grandma Doing? Predicting Functional Health Status from Binary Ambient Sensor Data. In 2012 AAAI Fall Symposium Series, pages 26-31, 2012.

[15] S. Robben, M. Pol, and B. Kröse. Longitudinal ambient sensor monitoring for functional health assessments: a case study. In Proceedings of the 2014 ACM International Joint Conference on Pervasive and Ubiquitous Computing: Adjunct Publication, pages 1209-1216. ACM, 2014.

[16] T. van Kasteren, A. Noulas, G. Englebienne, and B. Kröse. Accurate activity recognition in a home setting. In Proceedings of the 10th international conference on Ubiquitous computing, pages 1-9. ACM, 2008. 